\title{
The intravenous and oral pharmacokinetics of lotilaner in dogs
}

\author{
Céline E. Toutain*, Wolfgang Seewald and Martin Jung \\ Spanish version: Please see Additional file 1 (https://doi.org/10.1186/s13071-017-2475-z) for the Spanish version of this \\ research article and Additional file 2 for the French translation of the Abstract.
}

\begin{abstract}
Background: Lotilaner is a new oral ectoparasiticide from the isoxazoline class developed for the treatment of flea and tick infestations in dogs. It is formulated as pure S-enantiomer in flavoured chewable tablets (Credelio ${ }^{\mathrm{TM}}$ ). The pharmacokinetics of lotilaner were thoroughly determined after intravenous and oral administration and under different feeding regimens in dogs.
\end{abstract}

Methods: Twenty-six adult beagle dogs were enrolled in a pharmacokinetic study evaluating either intravenous or oral administration of lotilaner. Following the oral administration of $20 \mathrm{mg} / \mathrm{kg}$, under fed or fasted conditions, or intravenous administration of $3 \mathrm{mg} / \mathrm{kg}$, blood samples were collected up to 35 days after treatment. The effects of timing of offering food and the amount of food consumed prior or after dosing on bioavailability were assessed in a separate study in 25 adult dogs. Lotilaner blood concentrations were measured using a validated liquid chromatography/ tandem mass spectrometry (LC-MS/MS) method. Pharmacokinetic parameters were calculated by non-compartmental analysis. In addition, in vivo enantiomer stability was evaluated in an analytical study.

Results: Following oral administration in fed animals, lotilaner was readily absorbed and peak blood concentrations reached within 2 hours. The terminal half-life was 30.7 days. Food enhanced the absorption, providing an oral bioavailability above $80 \%$ and reduced the inter-individual variability. Moreover, the time of feeding with respect to dosing (fed $30 \mathrm{~min}$ prior, fed at dosing or fed 30 min post-dosing) or the reduction of the food ration to one-third of the normal daily ration did not impact bioavailability. Following intravenous administration, lotilaner had a low clearance of $0.18 \mathrm{l} / \mathrm{kg} /$ day, large volumes of distribution $V_{z}$ and $V_{s s}$ of 6.35 and $6.45 \mathrm{I} / \mathrm{kg}$, respectively and a terminal half-life of 24.6 days. In addition, there was no in vivo racemization of lotilaner.

Conclusions: The pharmacokinetic properties of lotilaner administered orally as a flavoured chewable tablet (Credelio ${ }^{\mathrm{TM}}$ ) were studied in detail. With a $\mathrm{T}_{\max }$ of $2 \mathrm{~h}$ and a terminal half-life of 30.7 days under fed conditions, lotilaner provides a rapid onset of flea and tick killing activity with consistent and sustained efficacy for at least 1 month.

Keywords: Lotilaner, Isoxazoline, Pharmacokinetics, Dog, Oral, Intravenous, Food effect, Fed, Fasted

\section{Background}

Infestation with fleas and ticks is an indisputable health challenge for dogs throughout the world as they can cause a variety of dermatological conditions and anaemia. Female fleas begin laying eggs within $24-36 \mathrm{~h}$ of acquiring a host, can produce as many as 40 to 50 eggs per day [1], rapidly creating an infestation. While the majority of 3host ticks infesting dogs are acquired from the external

* Correspondence: celine.toutain@elanco.com

Elanco Animal Health Inc., Mattenstrasse 24a, CH-4058 Basel, Switzerland environment and require additional hosts, Rhipicephalus sanguineus (sensu lato) (s.l.) prefers to feed on dogs during larval, nymphal and adult stages and will infest homes, kennels and veterinary hospitals. The R. sanguineus (s.l.) life-cycle can be completed entirely indoors in as little as 2 months under ideal conditions [2]. Additionally, fleas and ticks are competent vectors for numerous infectious diseases and secondary endoparasitic infections in dogs and vector-borne disease agents transmissible to people and other animal species [3]. Anecdotal reports of ectoparasite populations with reduced sensitivity to older flea and 
tick products are increasing [4]. Based on these issues, it is important to find new ways to rapidly address both fleas and ticks infestations.

The isoxazolines are a novel family of compounds shown to have activity against fleas and ticks [5-7]. Compounds from a library of over 1000 structures were screened against insects and acari in in vitro and in rodent studies. The lead candidate lotilaner, i.e. (S)-5-[5-(3,4,5-trichloro-phenyl)-5-trifluoromethyl-4,5-dihydroisoxazol-3-yl ]-3-methyl-thiophene-2-carboxylic acid [(2,2,2-trifluoroethylcarbamoyl)-methyl]-amide (Fig. 1) emerged as the most appropriate candidate when screened for both efficacy and safety. It has a molecular weight of 596.76, a measured log Pow (octanol/water partition coefficient) of 5.3. Lotilaner, as sarolaner [6] or afoxolaner [8], is highly bound to dog plasma proteins (unpublished data). With a chiral centre, $R$ - and $S$ - enantiomers can exist, the $S$ - enantiomer being lotilaner. As a difference in potency of 10100 fold between lotilaner and its opposite enantiomer $(R$ enantiomer) was measured in vitro against Ctenocephalides felis and R. sanguineus (s.l.) (unpublished data), Credelio $^{\mathrm{rm}}$ was developed as the pure $S$-enantiomer. This reduces the amount of active ingredient in the final formulation, eliminates the need for the inactive compound to be metabolised and/or excreted by the patient and limits the possibility of secondary pharmacodynamic effects. In other words, the same dose, if administered as pure $S$-enantiomer, provides better efficacy and longer protection period as compared to the racemate, provided that there is no in vivo racemization.

Isoxazolines are potent inhibitors of $\gamma$-aminobutyric acid (GABA)-gated chloride channels (GABACls) [9, 10]. The GABA-mediated chloride influx leads to hyperpolarization of the cellular membrane and generates an inhibitory postsynaptic potential, which decreases the probability of an action potential. Insects and other invertebrates possess GABACls that are expressed not only in the central nervous system, where they generate inhibitory potentials for the correct integration of neuronal signals but also at peripheral neuromuscular sites, where they promote muscular relaxation. In the presence of lotilaner, GABACls are

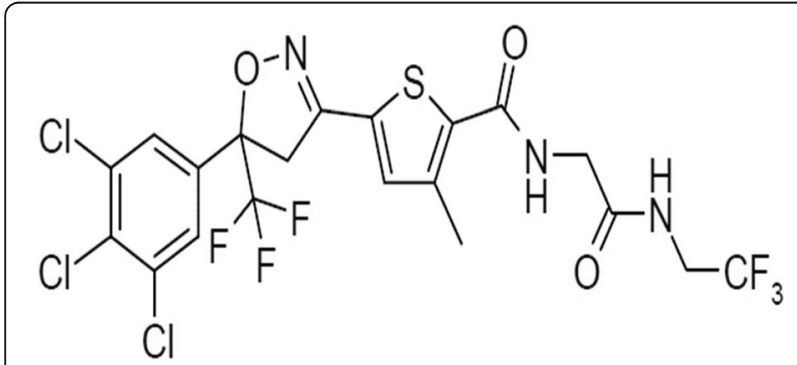

Fig. 1 Molecular structure of lotilaner ((S)-5-[5-(3,4,5-trichloro-phenyl)5-trifluoromethyl-4,5-dihydroisoxazol-3-yl] -3-methyl-thiophene-2carboxylic acid [(2,2,2-trifluoro-ethylcarbamoyl)-methyl]-amide) not able to open upon GABA stimulation, defining this molecule as an antagonist of GABACls. Parasites exposed to lotilaner endure a spastic paralysis leading to their starvation and death (unpublished data). In addition, isoxazolines are proven to be specific to insect and acari neuroreceptors, rather than mammalian neuroreceptors. The lack of effect on the mammalian nervous system at clinically relevant doses was confirmed in numerous laboratory and target animal safety studies. Repeated elevated doses administered orally at four-week intervals to young dogs at 8 weeks of age demonstrated that at the minimum dose rate of $43 \mathrm{mg} / \mathrm{kg} / \mathrm{month}$ lotilaner has a wide margin of safety [11].

At a dose of $20 \mathrm{mg} / \mathrm{kg}$, lotilaner has been shown to provide a rapid onset of activity against fleas and ticks that are sustained for at least 1 month following treatment [12-16]. Lotilaner is formulated as flavoured chewable tablets $\left(\right.$ Credelio $\left.^{\mathrm{Tm}}\right)$ and is to be administered orally on a monthly basis. To provide insights into the properties of lotilaner, support dose determination and formulation development, studies were performed in adult dogs to determine the pharmacokinetic profile after intravenous and oral administration, and to describe the effect of feeding on pharmacokinetic parameters.

\section{Methods}

\section{Animal management}

Purpose-bred mixed sex adult Beagle dogs and weighing approximately 9 to $16 \mathrm{~kg}$ were used. Each animal was uniquely identified and acclimatized to the study conditions for at least 1 week. Only healthy animals were included and suitability was evaluated by physical examination and clinical pathology. Dogs were housed indoors, in climate-controlled facilities in accordance with accepted laboratory animal care and use guidelines. They were kept in small groups except for the days around treatment administration where dogs were housed individually for at least 1 day, to avoid potential cross contamination between animals. Dogs were allowed the daily opportunity for outdoor exercise and social interaction. They were fed once daily with an appropriate ration of a commercial canine feed and water was available ad libitum. Dogs were observed for general health, behaviour and appetite at least once daily throughout the studies. All animals returned to their normal housing facilities on completion of the studies.

\section{Experimental designs \\ Study 1}

In the first study, designed to investigate the effect of feeding on lotilaner pharmacokinetics after oral administration, 25 mixed sex adult dogs were allocated to five treatment groups with five dogs in each group. Each dog received a single oral administration of a close-to-final 
tablet formulation, at the target dose of $15 \mathrm{mg} / \mathrm{kg}$ lotilaner (the initially intended therapeutic dose). Dogs were fasted overnight and five different feeding regimes were tested as follows: dogs received their full daily food allowance (i) 30 min prior to; (ii) at the same time; (iii) 30 min after; (iv) $5 \mathrm{~h}$ after treatment administration; or (v) only one-third of their full daily food allowance at the same time of treatment administration. Blood specimens were collected from the jugular vein in K3-EDTA tubes at pre-dose and at $30 \mathrm{~min}$, at 1, 2, 4, 8, 24, 48 and $72 \mathrm{~h}$ and at 7, 14 and 21 days post-treatment.

\section{Study 2}

In the second study, intended to determine the pharmacokinetic profile of lotilaner after intravenous and oral administration, 26 mixed sex adult dogs were allocated to three treatment groups as follows: one intravenous group of eight dogs, one oral group of 12 dogs treated $30( \pm 5)$ min after feeding, and one oral fasted group of six dogs. Each dog from the oral groups received a single administration of the final tablet formulation (containing $35 \% w / w$ lotilaner), at the target minimum dose of $20 \mathrm{mg} / \mathrm{kg}$ lotilaner, which was the final therapeutic dose. Each dog from the intravenous group received a single administration of lotilaner in a solution consisting of $23 \% \mathrm{w} / v$ physiological saline and tetraglycol ad 100\% $w /$ $v$, at the target dose of $3 \mathrm{mg} / \mathrm{kg}$ lotilaner, which was a dose known to be well tolerated intravenously. Blood specimens were collected from the jugular vein in K3EDTA tubes at pre-dose and at $5 \mathrm{~min}$ (intravenous only), $30 \mathrm{~min}$, at 1, 2, 4, 8, 24, 48 and $72 \mathrm{~h}$ and 7, 14, 21, 28 and 35 days post-treatment.

For both studies, whole blood specimens were stored frozen (for a maximum of 5 months) at approximately $-20{ }^{\circ} \mathrm{C}$ until analysis with a validated LC-MS/MS method. During validation, stability at storage conditions was demonstrated for at least 9 months.

\section{Analysis of lotilaner in blood}

Lotilaner was quantitatively analysed in blood using an analytical method involving liquid chromatography with tandem mass spectrometry detection (LC-MS/MS). Whole dog blood samples $(80 \mu \mathrm{l})$ were extracted by precipitation with acetonitrile and further diluted with acetonitrile. A proprietary closely related chemical analogue was used as the internal standard. Ten microliters of each diluted supernatant were chromatographed by HPLC on a reversed-phase column [Thermo Betasil C18, $5 \mu \mathrm{m}(50 \times 4.6 \mathrm{~mm})]$ with an isocratic mobile phase consisting of $0.1 \%$ formic acid and acetonitrile (15:85 v:v) using a flow rate of $0.8 \mathrm{ml} / \mathrm{min}$ and quantitatively analyzed on an AB Sciex API 5000 or API 5500 triple quadrupole mass spectrometer system using the negative Turbo IonSpray ionization mode and multiple reaction monitoring $(\mathrm{MRM})$ of the transition $\mathrm{m} / \mathrm{z} 596$ to 181 for lotilaner.

The method was validated over a linear range of 6.8 to $6800 \mathrm{ng} / \mathrm{ml}$, with a lower limit of quantification (LLOQ) of $6.8 \mathrm{ng} / \mathrm{ml}$, according to FDA and EMA guidelines [17, 18]. Mean inter-day precision was $14.9 \%$ at LLOQ and ranged between 3.4 and $7.8 \%$ at the other levels and the mean inter-day accuracy ranged between 100.3 and $103.6 \%$. In addition, specificity, dilution integrity, recovery and matrix effect, carryover, and stability in matrix and solutions were established. Long-term stability in frozen blood at $-20{ }^{\circ} \mathrm{C}$ was demonstrated over 9 months.

\section{Enantiomeric stability}

The in vivo enantiomeric stability of lotilaner was investigated in an analytical study. Blood specimens from 16 adult dogs which had received a single oral administration of the pure enantiomer drug at $15 \mathrm{mg} / \mathrm{kg}$ (tablet or chewy formulation, during an efficacy study) were analysed at four time points ( $4 \mathrm{~h}$ and 28, 56 and 84 days post-dosing) using an enantioselective analytical method. This method involved precipitation of $200 \mu \mathrm{l}$ whole blood with acetonitrile and subsequent solid phase extraction (SPE) on C18 cartridges, evaporation to dryness and reconstitution in heptane/ethanol 4:6, v/v. Enantiospecific analysis was carried out by chiral normal phase HPLC using a Daicel Chiralpak IA-3 column $(150 \times 4.6 \mathrm{~mm})$ and a mobile phase consisting primarily of heptane and isopropanol. Mass spectrometric detection was performed on an $A B$ Sciex API 4000 Qtrap triple quadrupole instrument using the negative Turbo IonSpray ionisation mode and multiple reaction monitoring (MRM).

\section{Pharmacokinetic and statistical analysis}

Pharmacokinetic parameters were calculated for individual animals using non-compartmental analysis. The validated statistical software $\mathrm{SAS}^{\odot}$, Version 9.2.2 was used for all calculations. The peak blood concentration $\left(C_{\max }\right)$ and time to peak concentration $\left(\mathrm{T}_{\max }\right)$ were observed values, for the oral groups. The terminal half-life $\left(T_{1 / 2 z}\right)$ was calculated by log-linear regression over a suitable time interval. The area under the concentration curve (AUC) between 0 and the last time point where the blood concentration was above the limit of quantitation $\left(\mathrm{AUC}_{\text {last }}\right)$, was calculated by the linear trapezoidal rule and values below the limit of quantitation at the beginning of the profile were treated as zero. The area under the concentration curve from zero to infinity ( $\left.A U C_{i n f}\right)$ was the sum of $\mathrm{AUC}_{\text {last }}$ and the extrapolation after the last observed timepoint; the second term was calculated by log-linear extrapolation from the last observed time point to infinity, using the half-life. The mean residence time (MRT) was calculated as the ratio of AUMC/AUC; where AUMC is the area under the first moment curve. 
The clearance per kilogram of body weight $(\mathrm{CL})$, defined as dose per kilogram of body weight/AUC, the volume of distribution at steady-state per kilogram of body weight $\left(\mathrm{V}_{\mathrm{ss}}\right)$, which is $\mathrm{CL} \times \mathrm{MRT}$ and the apparent volume of distribution per kilogram of body weight $\left(\mathrm{V}_{\mathrm{z}}\right)$, which is $C L \times T_{1 / 2 z} / \ln (2)$, were determined for the intravenous group only.

Bioavailability (F\%) in the oral groups was determined as (geometric mean of dose-normalized $\mathrm{AUC}_{\text {last }}$ in the oral group) / (geometric mean of dose-normalized $A U C_{\text {last }}$ in the intravenous group). In this study, $A U C_{\text {last }}$ was also equal to AUC from 0 to 35 days $\left(\mathrm{AUC}_{0-35 \mathrm{~d}}\right)$. $A U C_{\text {inf }}$ was found to be an unsuitable parameter for the evaluation of bioavailability because it was not accurate due to the high percentage extrapolated beyond the last measured data point.

A one-way analysis of variance (ANOVA) was performed on log-transformed dose-normalized $\mathrm{C}_{\max }$ and AUC parameters, with treatment as fixed effect. The mean and the $90 \%$ confidence interval (CI) for the difference between two treatment groups was calculated on the log scale and then back-transformed to the original scale, leading to the ratio between the two groups of $\mathrm{C}_{\max }$ or AUC. The difference (on the log scale) between two treatment groups can be tested versus zero in a ttest (degrees of freedom given in subscripted parentheses after the symbol $t$ in the tables; e.g. $t_{(21)}$ meaning a $t$-value with 21 degrees of freedom).

\section{Translations}

Spanish translation of the article is available in Additional file 1. French translation of the Abstract is available in Additional file 2.

\section{Results and discussion}

Enantiomeric stability in vivo

In 13 out of 16 adult dogs, no in vivo racemization was observed. In three dogs out of 16 animals, it was detectable only on day 84 but was negligible (less than 3\%) and is believed to be of no clinical relevance for safety or efficacy. The absence of in vivo racemization in dogs after administration of pure enantiomer of lotilaner was clearly demonstrated. The absence of in vivo racemization is a prerequisite for a pure enantiomeric drug to make sense and for the investigation of the pharmacokinetics and safety of the opposite enantiomer to be omitted.

\section{Effect of feeding in dogs}

Since food may influence the pharmacokinetics and as feeding may facilitate treatment administration by the dog owner, the effect of feeding (time and amount) was evaluated in detail. Drug concentrations versus time profiles under the five tested feeding regimes are shown in Fig. 2. A pronounced feeding effect was found for lotilaner, however the exact time of feeding with respect to dosing (fed $30 \mathrm{~min}$ prior, fed at dosing, fed $30 \mathrm{~min}$ postdosing) did not have a significant impact on bioavailability (see Table 1 for detailed test statistic and exact $P$ values). In addition, the reduction of the food ration to one-third of the daily ration also did not impact the bioavailability (Table 1 ). These findings offer a high degree of treatment flexibility to the dog owner, i.e. one-third of the daily ration is enough to provide adequate bioavailability and dosing can be performed at or around ( \pm $30 \mathrm{~min}$ ) the time of feeding. Hence, high bioavailability was found to be robust irrespective of changes in food

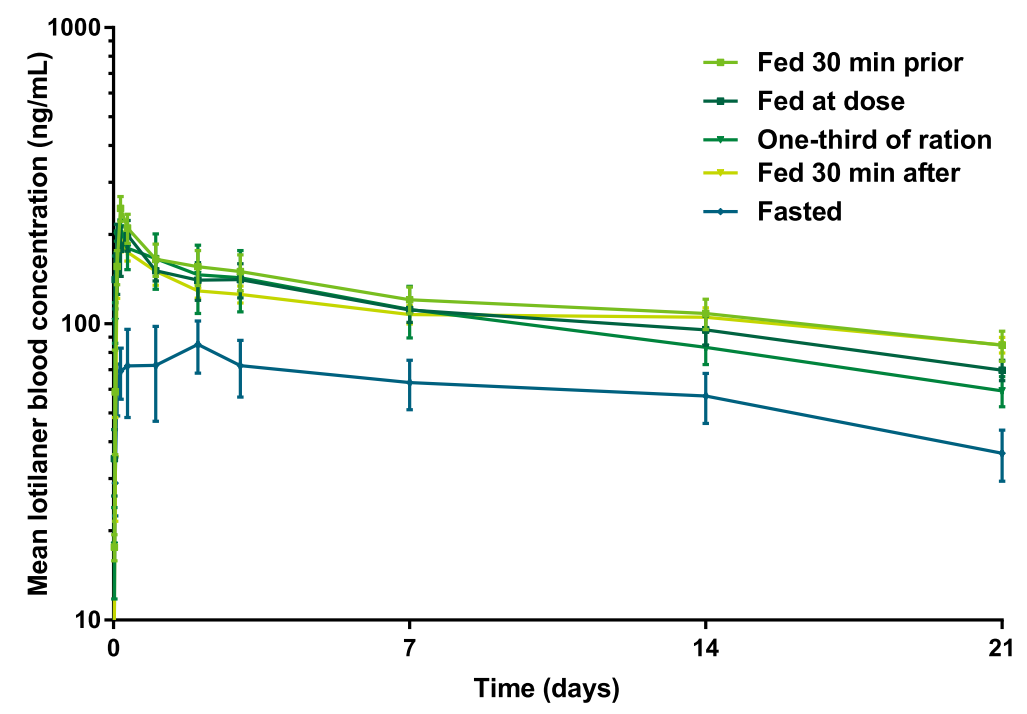

Fig. 2 Dose-normalized (to $15 \mathrm{mg} / \mathrm{kg}$ ) mean profile plots ( \pm standard error) of lotilaner following oral administration under different feeding regimes 
Table 1 Effect of different feeding regimens on bioavailability: statistical comparisons (Study 1)

\begin{tabular}{|c|c|c|c|c|c|}
\hline \multicolumn{2}{|l|}{ Treatment groups } & \multirow[t]{2}{*}{ Ratio $^{a}$ (first/second) } & \multirow[t]{2}{*}{$90 \% \mathrm{Cl}$} & \multirow[t]{2}{*}{$t$-value } & \multirow[t]{2}{*}{$P$-value } \\
\hline First & Second & & & & \\
\hline Fed 30 min prior & Fed at dose & 1.137 & $0.820-1.576$ & $t_{(20)}=0.68$ & 0.5052 \\
\hline Fed 30 min prior & Fed $1 / 3$ of ration & 1.252 & $0.904-1.735$ & $t_{(20)}=1.19$ & 0.2484 \\
\hline Fed 30 min prior & Fed 30 min after & 1.029 & $0.743-1.426$ & $t_{(20)}=0.15$ & 0.8799 \\
\hline Fed 30 min prior & Fasted & 2.250 & $1.624-3.118$ & $t_{(20)}=4.29$ & 0.0004 \\
\hline Fed at dose & Fed $1 / 3$ of ration & 1.101 & $0.795-1.526$ & $t_{(20)}=0.51$ & 0.6154 \\
\hline Fed at dose & Fed 30 min after & 0.905 & $0.653-1.255$ & $t_{(20)}=-0.53$ & 0.6050 \\
\hline Fed at dose & Fasted & 1.979 & $1.428-2.742$ & $t_{(20)}=3.61$ & 0.0018 \\
\hline Fed $1 / 3$ of ration & Fed 30 min after & 0.822 & $0.593-1.139$ & $t_{(20)}=-1.04$ & 0.3126 \\
\hline Fed $1 / 3$ of ration & Fasted & 1.797 & $1.297-2.490$ & $t_{(20)}=3.10$ & 0.0057 \\
\hline Fed 30 min after & Fasted & 2.186 & $1.577-3.029$ & $t_{(20)}=4.13$ & 0.0005 \\
\hline
\end{tabular}

${ }^{\mathrm{a}}$ Ratio of bioavailabilities i.e. ratio of $A U C_{\text {last }}$

${ }^{\mathrm{b}} \mathrm{P}$-values in bold denote significance

amount and exact timing; moreover, it was achievable with both dry and wet food (unpublished data). However, fasted conditions (fed $5 \mathrm{~h}$ post-dose) yielded significantly lower bioavailability (Table 1 ), similarly to what was observed for fluralaner [19]. High bioavailability together with low between-animal variability is crucial in order to ensure reliable and robust efficacy, as lotilaner is a systemically acting ectoparasiticide and consequently blood concentrations are expected to be directly correlated with efficacy. Any individual case of low bioavailability would be expected to translate into the lower duration of efficacy.

\section{Pharmacokinetic profile of lotilaner in dogs}

The pharmacokinetic parameters of lotilaner are summarised in Table 2 and drug concentrations versus time profiles after intravenous and oral administration under fed or fasted conditions are shown in Fig. 3. The actual dose in the intravenous group ranged from 3.08 to $3.24 \mathrm{mg} / \mathrm{kg}$, in the oral-fed group from 20.09 to $24.67 \mathrm{mg} / \mathrm{kg}$, and in the oral-fasted group from 20.16 to $24.62 \mathrm{mg} / \mathrm{kg}$. All pharmacokinetic parameters presented below are based on geometric means (considered as most appropriate, assuming that these parameters follow a log-normal distribution), except for $\mathrm{T}_{\max }$ which can

Table 2 Mean \pm standard deviation pharmacokinetic parameters of lotilaner in adult beagle dogs after either single administration at a target dose of $20 \mathrm{mg} / \mathrm{kg}$ orally to fasted dogs, orally to fed dogs, or at a target dose of $3.0 \mathrm{mg} / \mathrm{kg}$ intravenously

\begin{tabular}{|c|c|c|c|}
\hline Parameter & Intravenous, 3 mg/kg $(n=8)$ & Oral, fed $20 \mathrm{mg} / \mathrm{kg}(n=12)$ & Oral, fasted $20 \mathrm{mg} / \mathrm{kg}(n=6)$ \\
\hline Actual doses (mg/kg) & $3.08-3.24$ & $20.09-24.67$ & $20.16-24.62$ \\
\hline$T_{\max }$ (hours) & na & 2 (range: $1-24$ ) & 4 (range: $2-24$ ) \\
\hline$C_{\max }(\mathrm{ng} / \mathrm{ml})$ & na & $4011 \pm 990$ & $1454 \pm 849$ \\
\hline$A \cup C_{\text {last }}($ day*ng/ml) & $10,976 \pm 1381$ & $62,840 \pm 17,346$ & $18,592 \pm 16,632$ \\
\hline $\mathrm{AUC}_{\text {inf }}\left(\mathrm{day}^{*} \mathrm{ng} / \mathrm{ml}\right)$ & $17,844 \pm 1937$ & $118,600 \pm 42,287$ & $40,886 \pm 31,915$ \\
\hline$C_{\max }\left(\right.$ dose normalized $\left.{ }^{a}\right)(\mathrm{ng} / \mathrm{ml})$ & na & $179.2 \pm 43.5$ & $65.4 \pm 35.7$ \\
\hline$A \cup C_{\text {last }}\left(\right.$ dose normalized $\left.{ }^{a}\right)\left(\right.$ day $\left.^{*} \mathrm{ng} / \mathrm{ml}\right)$ & $3436 \pm 430$ & $2806 \pm 801$ & $837 \pm 731$ \\
\hline$A \cup C_{\text {inf }}\left(\right.$ dose normalized $\left.^{a}\right)\left(\right.$ day $^{*}$ ng/ml) & $5586 \pm 666$ & $5297 \pm 1921$ & $1840 \pm 1406$ \\
\hline $\mathrm{T}_{1 / 2 z}$ (day) & $24.6 \pm 5.9$ & $30.7 \pm 10.0$ & $38.8 \pm 11.2$ \\
\hline MRT (day) & $36.0 \pm 8.5$ & $45.3 \pm 13.9$ & $56.9 \pm 14.8$ \\
\hline CL (I/kg/day) & $0.18 \pm 0.02$ & na & na \\
\hline$V_{z}(1 / k g)$ & $6.35 \pm 1.27$ & na & na \\
\hline$V_{s s}(1 / k g)$ & $6.45 \pm 1.26$ & na & na \\
\hline Bioavailability (F\%) & na & 81.7 & 24.3 \\
\hline
\end{tabular}

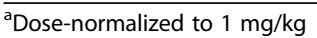

All values (Mean and standard deviation) are based on geometric summary statistics (corresponding to summary statistics of log transformed values and then back-transformed), except for $\mathrm{T}_{\max }$ which is based on the median 


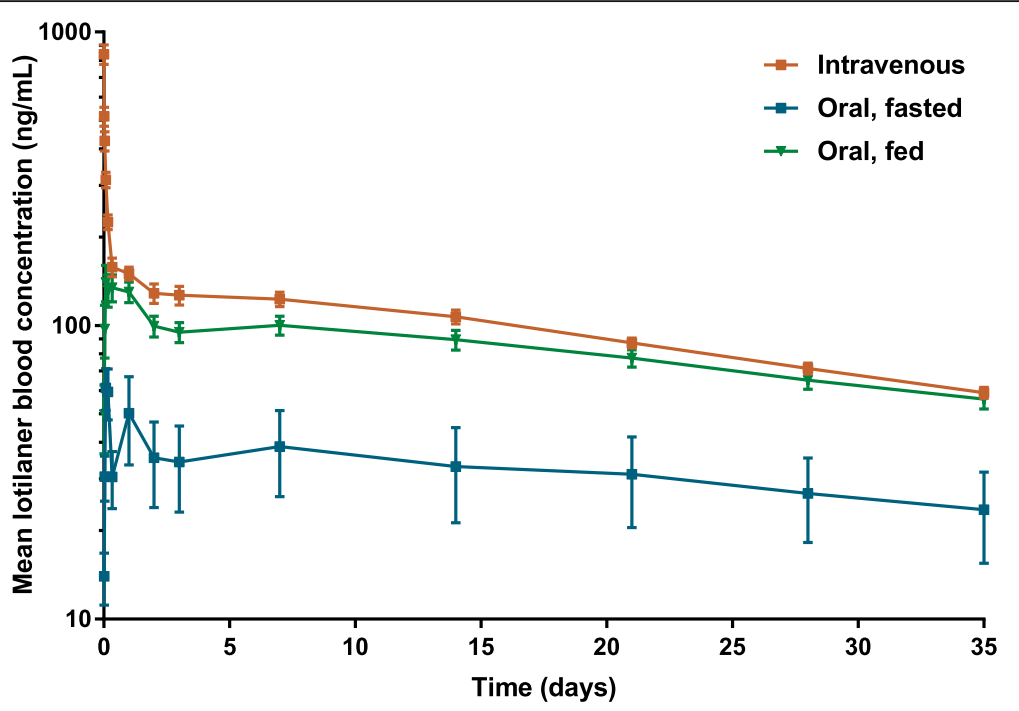

Fig. 3 Dose-normalized (to $1 \mathrm{mg} / \mathrm{kg}$ ) mean profile plots ( \pm standard error) of lotilaner following intravenous or oral administration to fed or fasted dogs

only take discrete values and is therefore based on the median.

After intravenous administration at $3 \mathrm{mg} / \mathrm{kg}$, a visual inspection of the profiles showed that lotilaner blood concentrations decreased bi-exponentially with a rapid distribution phase and a long elimination phase. The terminal half-life of lotilaner was 24.6 days and MRT was 36.02 days. Total blood clearance was $0.18 \mathrm{l} / \mathrm{kg} /$ day and the volumes of distribution $\mathrm{V}_{\mathrm{z}}$ and $\mathrm{V}_{\mathrm{ss}}$ were 6.35 and $6.45 \mathrm{l} / \mathrm{kg}$, respectively. Mean dose-normalized $\mathrm{AUC}_{\text {last }}$ was 3436 day*ng/ml.

After oral administration at $20 \mathrm{mg} / \mathrm{kg}$, a visual inspection of the profiles showed that lotilaner blood concentrations decreased bi-exponentially after $\mathrm{T}_{\max }$, with a rapid distribution phase within the first day of administration and a long elimination phase. After oral administration in fed conditions, detectable blood levels were identified in most treated dogs within $30 \mathrm{~min}$ and concentrations peaked quickly (mean dose-normalized $C_{\max }$ of $179 \mathrm{ng} /$ $\mathrm{ml}$ ) with a $\mathrm{T}_{\max }$ at $2 \mathrm{~h}$, indicating rapid dissolution and absorption of the chewable tablet. The terminal half-life was 30.7 days and MRT was 45.3 days. Mean dose-normalized $\mathrm{AUC}_{\text {last }}\left(=\mathrm{AUC}_{0-35 \mathrm{~d}}\right)$ was 2806 day*ng/ml. After oral administration in fasted conditions, lower blood concentrations of lotilaner were observed with a mean dosenormalized $\mathrm{C}_{\max }$ of $65 \mathrm{ng} / \mathrm{ml}$. $\mathrm{T}_{\max }$ was observed later, at $4 \mathrm{~h}$. The terminal half-life was 38.7 days and MRT was 56.9 days. Mean dose-normalized $\mathrm{AUC}_{\text {last }}\left(=\mathrm{AUC}_{0-35 \mathrm{~d}}\right.$ ) was 837 day*ng/ml. The mean terminal half-life after oral administration was in the same range as the one determined after intravenous administration, indicating that the terminal phase represented the true elimination phase.

For comparisons from the oral-fed and oral-fasted groups, differences in mean values for $\mathrm{C}_{\max }$, $\mathrm{AUC}_{\text {last }}$,
$\mathrm{AUC}_{\text {inf }}$ were significant and the difference in bioavailability (Table 2) between the oral-fed (81.7\%) and oralfasted groups $(24.3 \%)$ was also significant (see Table 3 for detailed test statistic and exact $P$-values). The differences in half-life and mean residence time between fed and fasted states were not significant (Table 3). Moreover, the variability of the lotilaner pharmacokinetic parameters was moderate in the intravenous and oral-fed groups and much higher in the oral-fasted group. This high variability in the oral-fasted group was explained by the low bioavailability since this is known as a major source of variability [20].

In order to interpret the clearance, the overall body extraction ratio (which can be regarded as the percentage of drug being cleared by the entire body during a single passage through the clearing organs) was computed by the body clearance $(0.18 \mathrm{l} / \mathrm{kg} /$ day $)$ divided by the cardiac output (approximately $167 \mathrm{l} / \mathrm{kg} /$ day for a $10 \mathrm{~kg}$ dog) [21]. Hence, the total blood clearance corresponded to an overall extraction ratio of $0.1 \%$ and is therefore considered as very low. In addition, lotilaner had high volumes of distribution ( $>6 \mathrm{l} / \mathrm{kg}$ ), as expected for a lipophilic drug that would distribute in the fatty tissue. The low clearance combined with the large volume of distribution explains the long half-life of lotilaner in the $\operatorname{dog}[22,23]$. As compared to the other isoxazolines developed for dogs (afoxolaner, fluralaner and sarolaner), lotilaner in the present study had the longest half-life (approximately 4 weeks versus 2 weeks for the other compounds). This difference was mainly explained by the largest volume of distribution (approximately $6 \mathrm{l} / \mathrm{kg}$ for lotilaner versus $3 \mathrm{l} / \mathrm{kg}$ for the other compounds), whereas the clearance was in the same range (from 0.12 to $0.18 \mathrm{l} / \mathrm{kg} /$ day) $[6,8,24]$. This long terminal half-life 
Table 3 Comparisons between fed and fasted groups following oral administration of lotilaner at 20 mg/kg (Study 2)

\begin{tabular}{|c|c|c|c|c|}
\hline Parameter & Ratio (fasted to fed) & $90 \% \mathrm{Cl}$ & $t$-value & $P$-value ${ }^{a}$ \\
\hline$T_{1 / 2 z}$ & 1.263 & $0.983-1.624$ & $t_{(23)}=-1.59$ & 0.1244 \\
\hline MRT & 1.256 & $0.991-1.591$ & $t_{(23)}=-1.65$ & 0.1128 \\
\hline$C_{\max }($ dose normalized) & 0.365 & $0.265-0.502$ & $t_{(16)}=5.51$ & $<0.0001$ \\
\hline$A \cup C_{\text {last }}$ (dose normalized) & 0.298 & $0.201-0.441$ & $t_{(23)}=5.29$ & $<0.0001$ \\
\hline$A \cup C_{\text {inf }}($ dose normalized) & 0.347 & $0.238-0.507$ & $t_{(23)}=4.80$ & $<0.0001$ \\
\hline Bioavailability & 0.298 & $0.201-0.441$ & $t_{(23)}=5.29$ & $<0.0001$ \\
\hline
\end{tabular}

${ }^{a} P$-values in bold denote significance

and mean residence time explains the persistent systemic availability of lotilaner and provides effective blood concentrations for the entire duration of the interdosing interval of 1 month. Variability in half-life between individuals or between studies and populations was observed in the numerous studies performed during the development program, however, care was taken during dose characterization to select a robust dose high enough to provide 1 month duration of efficacy even in individuals with a shorter half-life and at the lowest possible therapeutic dose within the dose band.

The feeding effect on pharmacokinetics was multiple, not only did administration in fasted dogs lead to much lower bioavailability, but also to a delayed $\mathrm{T}_{\max }$ and to an increase between-animal variability. Consequently, administration of lotilaner to fasted dogs is not recommended. Achievement of the maximum blood concentration within $2 \mathrm{~h}$ following lotilaner administration to dogs in the fed state aligns with the demonstrated rapid onset of adulticidal (flea and tick) activity [12-16]. Similarly, the demonstrated long half-life of lotilaner (30.7 days in the oral-fed group) and the sustained concentration levels above the estimated flea- and ticklethal breakpoints through at least 1 month align with the prolonged effectiveness observed in multiple studies in which challenge with these parasites was extended through 35 days after lotilaner treatment.

\section{Conclusions}

The pharmacokinetic properties of lotilaner $\left(\right.$ Credelio $\left.^{\mathrm{Tm}}{ }^{\mathrm{W}}\right)$ were investigated in a number of studies. Following a single intravenous administration, lotilaner had a very low clearance $(0.18 \mathrm{l} / \mathrm{kg} / \mathrm{day})$ and high volumes of distribution (> $6 \mathrm{l} / \mathrm{kg}$ ), resulting in a long terminal half-life (24.6 days). Following a single oral administration to fed dogs, lotilaner blood concentrations peaked within 2 hours, had a long half-life of 30.7 days, and had significantly greater bioavailability than when administered to fasted dogs. Food (at least $1 / 3$ of the daily ration) enhanced the bioavailability to $>80 \%$ and therefore it is recommended to administer chewable flavoured tablets at or around the time of feeding. Hence, lotilaner administered to fed dogs at a minimum dose of $20 \mathrm{mg} / \mathrm{kg}$ should, therefore, provide a rapid onset of flea and tick killing activity, with consistent and sustained effectiveness for at least 1 month after treatment.

\section{Additional files}

Additional file 1: Spanish translation of the article. (PDF $148 \mathrm{~kb}$ )

Additional file 2: French translation of the Abstract. (PDF $17 \mathrm{~kb}$ )

\section{Abbreviations}

ANOVA: Analysis of variance; $\mathrm{AUC}_{\text {inf: }}$ Area under the blood concentrationtime curve from zero to infinity; $A \cup C_{\text {last }}$ : Area under the blood concentration-time curve from zero to the last time point for which blood concentration is above the limit of quantitation; $\mathrm{Cl}$ : Confidence interval; $\mathrm{CL}$ : Total body clearance of drug from the blood; $\mathrm{C}_{\text {max }}$ : Maximum (peak) blood drug concentration; EMA: European Medicine Agency; FDA: Food and Drug Administration; GABACls: $\gamma$-aminobutyric acid (GABA)-gated chloride channels; LC-MS/MS: Liquid chromatography tandem mass spectrometry; LLOQ: Lower limit of quantification; na: Not applicable; $T_{1 / 2 z}$ : Elimination halflife associated with the terminal slope of a semi-logarithmic concentration time curve; $T_{\text {max }}$ : Time to reach maximum (peak) blood concentration following drug administration; $V_{\text {ss: }}$ Volume of distribution at steady-state; $V_{z}$ : Volume of distribution during the terminal phase

\section{Acknowledgements}

The authors would like to thank all personnel involved in the studies.

Funding

All studies were funded by Elanco.

Availability of data and materials

Not applicable

Authors' contributions

CET drafted the manuscript and all authors revised and approved the final version. MJ authored the study designs, WS performed the pharmacokinetic analysis and all authors participated in the result interpretation and study completion. All authors read and approved the final manuscript.

\section{Ethics approval and consent to participate}

For the two studies, animal procedures were conducted in compliance with respective animal welfare national legislation and approved by relevant ethical committees. The first study (Study 1) was conducted in Australia, approved by the test site's Animal Ethics Committee and conducted under an Australian Pesticides and Veterinary Medicines Authority (APVMA) trial permit. The second study (Study 2) was conducted in Switzerland, approved by the company's Animal Welfare Officer and conducted under an animal experimentation permit from the Swiss cantonal authorities.

Consent for publication

Not applicable

Competing interests

CET, WS and MJ are employees of Elanco. 


\section{Publisher's Note}

Springer Nature remains neutral with regard to jurisdictional claims in published maps and institutional affiliations.

Received: 14 December 2016 Accepted: 12 October 2017

Published online: 01 November 2017

\section{References}

1. Rust MK. The biology, ecology and management of the cat flea. Annu Rev Entomol. 1997:452:451-73.

2. Bowman DD. Georgis' parasitology for veterinarians. St Louis: Elsevier Health Sciences; 2014

3. Jongejan F, Uilenberg G. The global importance of ticks. Parasitology. 2004; 129(Suppl):3-14

4. Coles TB, Dryden MW. Insecticide/acaricide resistance in fleas and ticks infesting dogs and cats. Parasit Vectors. 2014;7:8.

5. Shoop WL, Hartline EJ, Gould BR, Waddell ME, McDowell RG, Kinney JB, et al. Discovery and mode of action of afoxolaner, a new isoxazoline parasiticide for dogs. Vet Parasitol. 2014;201:179-89.

6. McTier TL, Chubb N, Curtis MP, Hedges L, Inskeep GA, Knauer CS, et al. Discovery of sarolaner: a novel, orally administered, broad-spectrum, isoxazoline ectoparasiticide for dogs. Vet Parasitol. 2016;222:3-11.

7. Gassel M, Wolf C, Noack S, Williams H, Ilg T. The novel isoxazoline ectoparasiticide fluralaner: selective inhibition of arthropod $\gamma$-aminobutyric acid- and L-glutamate-gated chloride channels and insecticidal/acaricidal activity. Insect Biochem Mol Biol. 2014;45:111-24.

8. Letendre L, Huang R, Kvaternick V, Harriman J, Drag M, Soll M. The intravenous and oral pharmacokinetics of afoxolaner used as a monthly chewable antiparasitic for dogs. Vet Parasitol. 2014;201:190-7.

9. Ozoe Y, Asahi M, Ozoe F, Nakahira K, Mita T. The antiparasitic isoxazoline A1443 is a potent blocker of insect ligand-gated chloride channels. Biochem Biophys Res Commun. 2010;391:744-9.

10. Lahm GP, Cordova D, Barry JD, Pahutski TF, Smith BK. Long JK, et al. 4Azolylphenyl isoxazoline insecticides acting at the GABA gated chloride channel. Bioorg Med Chem Lett. 2013;23:3001-6.

11. Kuntz E, Kammanadiminti S. Safety evaluation of lotilaner in dogs after ora administration as flavoured chewable tablets $\left(\right.$ Credelio $\left.^{\mathrm{TM}}\right)$. Parasit Vectors. 2017. (In press).

12. Cavalleri D, Murphy M, Seewald W, Drake J, Nanchen S. Assessment of the speed of flea kill of lotilaner (Credelio ${ }^{\mathrm{TM}}$ ) throughout the month following oral administration to dogs. Parasit Vectors. 2017. (In press).

13. Cavalleri D, Murphy M, Seewald W, Drake J, Nanchen S. Assessment of the onset of lotilaner (Credelio ${ }^{\mathrm{TM}}$ ) speed of kill of fleas on dogs. Parasit Vectors. 2017. (In press).

14. Murphy M, Cavalleri D, Seewald W, Drake J, Nanchen S. Laboratory evaluation of the speed of kill of lotilaner $\left(C\right.$ redelio $\left.{ }^{T M}\right)$ against Ixodes ricinus ticks on dogs. Parasit Vectors. 2017. (In press).

15. Karadzovska D, Chappell K, Coble S, Murphy M, Cavalleri D, Wiseman S, et al. A randomized, controlled field study to assess the efficacy and safety of lotilaner flavored chewable tablets $\left(\right.$ Credelio $^{\text {TM }}$ ) in eliminating fleas in clientowned dogs in the USA. Parasit Vectors. 2017. (In press).

16. Cavalleri D, Murphy M, Seewald W, Drake J, Nanchen S. A randomized, blinded, controlled field study to assess the efficacy and safety of lotilaner $\left(\right.$ Credelio $\left.^{\mathrm{TM}}\right)$ in controlling fleas in client-owned dogs in Europe. Parasit Vectors. 2017. (In press).

17. FDA. Guidance for industry, bioanalytical method validation. Rockville: U.S. Department of Health and Human Services, Food and Drug Administration, Center for Drug Evaluation and Research (CDER), Center for Veterinary Medicine (CVM); 2001.

18. EMEA: Guideline on bioanalytical method validation, the European Agency for the Evaluation of medicinal products/Committee for Medicinal Products for human use, EMEA/CHMP/EWP/192217/2009, 2011.

19. Walther FM, Allan MJ, Roepke RKA, Nuernberger MC. The effect of food on the pharmacokinetics of oral fluralaner in dogs. Parasit Vectors. 2014;7:84.

20. Toutain PL, Bousquet-Mélou A. Bioavailability and its assessment. J Vet Pharmacol Ther. 2004;27:455-66.

21. Toutain PL, Bousquet-Mélou A. Clearance. J Vet Pharmacol Ther. 2004 $27: 415-25$.
22. Toutain PL, Bousquet-Mélou A. Volumes of distribution. J Vet Pharmacol Ther. 2004;27:441-53.

23. Toutain PL, Bousquet-Mélou A. Plasma terminal half life. J Vet Pharmacol Ther. 2004:27:427-39.

24. Kilp S, Ramirez D, Allan MJ, Roepke RK, Nuernberger MC. Pharmacokinetics of fluralaner in dogs following a single oral or intravenous administration. Parasit Vectors. 2014;7:85.

\section{Submit your next manuscript to BioMed Central and we will help you at every step:}

- We accept pre-submission inquiries

- Our selector tool helps you to find the most relevant journal

- We provide round the clock customer support

- Convenient online submission

- Thorough peer review

- Inclusion in PubMed and all major indexing services

- Maximum visibility for your research

Submit your manuscript at www.biomedcentral.com/submit
) Biomed Central 